\title{
Paraneoplastic syndromes in daily clinical practice
}

\author{
Monika Wójtowicz' , Agata Rękas-Wójcik', Aleksandra Lipa², Andrzej Prystupa', Grzegorz Dzida' \\ ${ }^{1}$ Department of Internal Medicine, Medical University, Lublin, Poland \\ ${ }^{2}$ Children's Regional Hospital of St Louis, Cracow, Poland \\ Wójtowicz M, Rękas-Wójcik A, Lipa A, Prystupa A, Dzida G. Paraneoplastic syndromes in daily clinical practice. J Pre-Clin Clin Res. 2014; 8(2): \\ 71-75. doi: 10.5604/18982395.1135653
}

\begin{abstract}
Paraneoplastic syndromes consist of disorders that accompany benign and malignant tumours, but are not directly related to mass effects or invasion by the primary tumour or its metastases. The pathophysiology of mostly paraneoplastic syndromes is not well known. The usual mechanism of their development is the aberrant production of substances (protein hormones, hormone precursors or hormone-like substances) by tumour tissue or autoimmune disorder. The neurological paraneoplastic syndromes are autoimmune disorders that can affect almost any part of the nervous system. The endocrine paraneoplastic syndromes are often certain clinical syndromes like paraneoplastic hypercalcaemia or the aberrant production of hormones by cancers. Many haematologic conditions, including anaemia, leucocytosis, thrombocytopenia or thrombocytosis, coagulapathy have been reported in association with cancer. Also paraneoplastic rheumatic syndromes have been reported in association of cancer. Skin can be involved in cancer in different ways: by metastases, as a part of a genetic disorder with a cutaneous component in which there is an inherited predisposition to the later development of malignancy, as a part of an acquired syndrome due to the toxicity of a carcinogen that induces malignant change and accompanying skin changes, as a consequence of immunosuppression, or by the development of specific lesions that occur as a paraneoplastic syndromes The most frequently diagnosed dermatologic conditions include acrokeratosis paraneoplastica, Sweet's syndrome and paraneoplastic pemphigus. The purpose of this article is to describe the most popular in internal clinical practice neurologic, endocrine, haematologic, rheumatologic and dermatologic paraneoplastic syndromes associated with cancer.
\end{abstract}

\section{Key words}

Paraneoplastic syndromes, cancer, clinical practice

\section{INTRODUCTION}

It is assumed that $10 \%$ of hospitalized patients with cancer develop paraneoplastic syndromes of different clinical presentations, mainly neurologic, endocrine, haematologic, rheumatologic and dermatologic.

The most common paraneoplastic syndromes are neurologic conditions, including paraneoplastic encephalomyelitis, Lambert-Eaton myasthenic syndrome, subacute sensory neuronopathy, subacute cerebellar degeneration, limbic encephalitis, opsoclonus/ataxia and autonomic neuronopathy. The endocrine paraneoplastic syndromes are often certain clinical syndromes, such as paraneoplastic hypercalcaemia, the syndrome of inappropriate secretion of antidiuretic hormone or the aberrant production of other hormones by cancers. Many haematological conditions, including anaemia (haemolytic anaemia or anaemia of chronic disease), leucocytosis, thrombocytopenia or thrombocytosis, coagulapathy (venous thromboembolism, Trousseau's syndrome, disseminated intravasculer coagulation (DIC)) have been reported in association with cancer. Also, paraneoplastic rheumatic syndromes comprise hypertrophic osteoarthropathy, polyarthritis, dermatomyositis/polymyositis and paraneoplastic vasculitis which have been reported in association of cancer. The most frequently diagnosed dermatologic conditions include acrokeratosis paraneoplastica, Sweet's syndrome (acute febrile neutrophilic dermatosis) and paraneoplastic pemphigus.

The paraneoplastic syndromes occur most commonly in patients with small-cell lung carcinoma, urinary system

Address for correspondence: Andrzej Prystupa, Chair and Department of Internal Medicine, Medical University, Staszica 16, 20-081 Lublin, Poland

E-mail: aprystup@mp.pl

Received: 27 October 2012; accepted: 22 September 2014 and gynecological carcinomas, lymphomas, malignant melanoma, breast cancer, thymoma and mesenchymal tumours.

An overview of the literature demonstrates that the diagnostic criteria and laboratory markers for some of the paraneoplastic syndromes are helpful in daily clinical practice.

Paraneoplastic neurological syndromes. The neurological paraneoplastic syndromes are rare disorders and are observed in less than $1 \%$ of solid tumours, $3 \%$ for small cell lung cancer and up to $15 \%$ for thymoma patients [1].

Usually, neurological problems in oncologic patients are representd by the mass effects or invasion by the primary tumour or its metastases in the nervous system. It is well recognized that treatments designed to kill cancer cells may be neuro-toxic - including damage to the nervous system from chemotherapy and radiotherapy [2]. Paraneoplastic neurological syndromes (PNS) are autoimmune disorders, and antibodies in serum and cerebrospinal fluid (CSF) are observed that recognize the antigenes shared by neurons and tumour cells [3].

The neurological paraneoplastic syndromes are disorders that can affect almost any part of the nervous system. An identical syndrome can also occur in patients without cancer. Clues that a patient's symptoms could be caused by PNS include an acute or subacute onset, a progressive course leading to severe disability, inflammatory changes in the CSF, and the presence of an antibody directed against a neural antigen. Patients with PNS frequently exhibit serum's and CSF's antibody responses directed against neurons, often with oligoclonal bands [2,4]. Patients with PNS may be divided into several groups on their antigenic targets. The neurological paraneoplastic syndromes are certain 
clinical syndromes, such as paraneoplastic encephalomyelitis (PEM), Lambert-Eaton myasthenic syndrome (LEMS), subacute sensory neuronopathy (SSN), subacute cerebellar degeneration, limbic encephalitis, opsoclonus/ataxia, and autonomic neuronopathy [5].

Involvement of the cranial nerves is one of the manifestations of anti-Hu-associated brainstem encephalitis [6], which is associated with a small-cell lung carcinoma in the majority of patients [7]. The diagnosis of anti-Hu-associated encephalomyelitis/sensory neuropathy may be particularly difficult when the cranial nerve's involvement represents the first clinical manifestation of the disease. A case has been reported of a patient [7] who presented with facial pain as the first manifestation of an anti-Hu paraneoplastic syndrome.

It is important that paraneoplastic neurological syndromes can be associated with autoantibodies to the $\mathrm{Hu}$ antigen which is cross-reactive with the Sjogren SS-A antigen, and with the rheumatic disease-associated antigens anti-U1ribonucleoprotein, Anti-Smith (Anti-Sm) antibodies (RNP, Sm) [7].

Most cases of Guillain-Barre syndrome (GBS) are preceded by respiratory or gastrointestinal infections or immunization. GBS has also been linked to malignancies as a possible paraneoplastic complication. Tumours associated with GBS are lymphomas, breast, small-cell lung, renal and oesophageal carcinomas [8].

Paraneoplastic endocrine syndromes. The paraneoplastic endocrine syndromes include a varied group of symptoms associated with cancer. Nearly all the diseases are associated with the production of peptide hormones which have autocrine stimulatory effects [9]. The symptoms are not related to the local effects of the cancers or to their distant spread, but are the result of the production of hormones, growth factors, cytokines or other substances by the tumour cells [23].

The first information of endocrine syndromes in patients with non-endocrine neoplasm dates from the 1920s, and the idea of hormone production by non-endocrine cancers was first published in 1941. The patient was 51-year-old man with a tumour in the right ilium with a hypercalcaemia. A biopsy of the bone lesion exposed metastatic renal cell carcinoma. The patient was treated by radiotherapy and the calcium level in the serum decreased [9].

Hypercalcaemia is the most common paraneoplastic endocrine syndrome and approximately $5 \%$ of patients with malignant tumors will develop hypercalcaemia [23], in other clinical trials in nearly $10 \%$ of cases [10]. In hospitalized patients with hypercalcaemia, about $25 \%$ will have an underlying malignancy [24]. Hypercalcaemia is defined as the level of serum calcium above the upper limit of normal range $-2.58 \mathrm{mmol} / 1$ for men, $2.50 \mathrm{mmol} / 1$ for women aged under 50 , and $2.56 \mathrm{mmol} / \mathrm{l}$ for women above 50 years of age [10]. Hypercalcaemia in these patients usually has a rapid clinical beginning and patients may present confusion, nausea, vomiting and dehydration. Non-small cell carcinomas of the lung, breast cancer, and cancer of the head and neck, esophagus, urinary system and gynecological carcinomas, multiple myeloma and lymphoma, are the most common underlying cancers. Prognosis is poor for most patients [9].

The syndrome of inappropriate secretion of antidiuretic hormone (SIADH) is the second most common paraneoplastic endocrine syndrome [23]. Laboratory findings include hyponatraemia (serum sodium less than $130 \mathrm{mmol} / \mathrm{L}$ ) and inappropriately increased urine osmolarity. The SIADH occurs most commonly in patients with smallcell bronchogenic carcinoma (15\%), and has also been reported in patients with neuroendocrine tumours in a variety of other sites, including the cervix, prostate, head and neck malignancies (3\%), non-small-cell bronchogenic carcinoma $(0.3 \%)$, and in patients with a wide variety of other malignancies involving the central nervous system, uterus and ovaries, breast, skin, thorax and soft tissues [9]. The vasopressin level increases in about $50 \%$ of patients with small-cell bronchogenic carcinoma, but SIADH occurs in only $15 \%$ [25]. The atrial natriuretic peptide (ANP) has also been associated in the progress of some cases of tumour with hyponatraemia [26], and both ANP and vasopressin can be produced by the same cancer cells [27].

The paraneoplastic production of ACTH occurs in nearly $15 \%$ of cases of Cushing's syndrome, while CRH production is responsible for a small group of patients [23]. Small-cell carcinomas of the lung account for at least $50 \%$ of cases of the syndrome. Other neuroendocrine tumours, including bronchial and thymic carcinoids, pancreatic endocrine tumours, pheochromocytomas and medullary thyroid carcinomas, are responsible for most of the remaining cases [9].

The acromegaly associated with non-pituitary cancers is rare. The paraneoplastic production of growth hormone associated with acromegaly is extraordinary and has been documented in patients with lung carcinoma, pancreatic endocrine tumour and non-Hodgkin's lymphoma. Tumors usually associated with the production of growth hormonereleasing hormone GHRH include pancreatic endocrine tumours, carcinoids, small-cell lung carcinomas and pheochromocytomas. The paraneoplastic production of growth hormone occurs more frequently in pancreatic (25\%) than in gastrointestinal (11\%) endocrine tumours [9].

Tumours associated with human chorionic gonadotropin (hCG) production may occur in association with nontrophoblastic and non-germ-cell tumours, including pulmonary, gastrointestinal, hepatocellular, gynecological and genitourinary malignancies. Clinically, the production of hCG may be associated with gynecomastia in men [9].

The production of insulin-like growth factor II (IGF-II) is responsible for the many of cases of hypoglycaemia associated with extra-pancreatic tumou, they are generally large and usually types of sarcoma. Hepatocellular carcinomas, adrenocortical carcinomas, carcinoid tumours, leukemias and lymphomas, can also be responsible for hypoglycaemia. It hardly ever happens that insulin production may be responsible for hypoglycaemia in oncologic patients [9].

Osteogenic hypophosphatemic osteomalacia is a rare paraneoplastic syndrome [23], characterized by decreased mineralization of newly-formed bone and by the clinical findings of osteomalacia. Muscle weakness and bone fractures are frequent. Laboratory findings include marked hypophosphataemia, increased levels of alkaline phosphatase and normal levels of calcium and parathyroid hormone. The level of 25-hydroxyvitamin D is normal, while $1,25\left(\mathrm{OH}_{2}\right) \mathrm{D}$ is usually low. The syndrome has been generally associated with mesenchymal tumours, and rarely carcinomas of the lung and prostate [9].

The paraneoplastic production of renin is a rare endocrine manifestation of malignant tumours. Overload of renin production is associated with the hypertension and the hypokalaemia. Cancers with renin production include: 
Table 1. Major neoplasms associated with paraneoplastic syndromes $[1,3,17,18,23,26]$

\begin{tabular}{|c|c|c|}
\hline & PARANEOPLASTIC SYNDROMES & MAJOR ASSOCIATED NEOPLASMS \\
\hline \multirow{5}{*}{$\begin{array}{l}\text { Paraneoplastic } \\
\text { neurological } \\
\text { syndromes }\end{array}$} & Cerebellar degeneration & Gynaecologic and breast neoplasms; Hodgkin's disease \\
\hline & Encephalomyeloneuritis & Small-cell carcinoma; neuroendocrine tumours; myxoid chondrosarcoma \\
\hline & Opsoclonus-myoclonus & Breast cancer; small-cell lung carcinoma \\
\hline & Limbic encephalitis & Hodgkin's disease \\
\hline & Myasthenia gravis & Thymoma \\
\hline \multirow{6}{*}{$\begin{array}{l}\text { Paraneoplastic } \\
\text { endocrine } \\
\text { syndromes }\end{array}$} & Hypercalcaemia & $\begin{array}{l}\text { Squamous cell carcinoma of lung, skin, head and neck; renal carcinoma; myeloma; small-cell carcinoma of } \\
\text { lung; ovarian carcinoma; thymoma; breast carcinomas; other solid tumors; multiple myeloma }\end{array}$ \\
\hline & $\begin{array}{l}\text { Syndrome of inappropriate antidiuretic } \\
\text { hormone secretion (SIADH) }\end{array}$ & Small-cell carcinoma of lung, prostate, other sites; adenocarcinoma of prostate, pancreas, etc. \\
\hline & Cushing's syndrome & $\begin{array}{l}\text { Small-cell carcinoma of lung, and other sites; pancreatic endocrine tumor; medullary thyroid carcinoma; } \\
\text { hypothalamic gangliocytoma; bronchial carcinoid; pheochromocytoma }\end{array}$ \\
\hline & Gynecomastia & $\begin{array}{l}\text { Small-cell carcinoma of lung; adenocarcinoma of lung; pheochromocytoma; hepatoma; gastrointestinal } \\
\text { tumor; germ cell tumour; breast carcinoma; carcinoma of prostate, ovary, cervix, adrenal, bladder, liver; } \\
\text { osteogenic sarcoma; lymphoma }\end{array}$ \\
\hline & Hypoglycaemia & Sarcoma; Wilm's tumor; hepatocellular carcinoma \\
\hline & Zollinger-Ellison syndrome & Pancreatic endocrine tumour; tumours of other sites \\
\hline \multirow{4}{*}{$\begin{array}{l}\text { Paraneoplastic } \\
\text { haematologic } \\
\text { syndromes }\end{array}$} & Venous thromboembolism (VTE) & Haematologic malignancies; solid tumours (brain, lung, uterus, bladder, pancreas, stomach, kidney) \\
\hline & Trousseau's syndrome & Haematologic malignancies; solid tumours \\
\hline & Anaemia & Hematologic malignancies; solid tumors \\
\hline & Leukocytosis & Haematologic malignancies; squamous cell carcinoma \\
\hline \multirow{3}{*}{$\begin{array}{l}\text { Paraneoplastic } \\
\text { rheumatic } \\
\text { syndromes }\end{array}$} & Arthritis & Lung cancer; breast, colon, ovarian cancers; gastric and oesophageal cancers; lymphoprolipherative diseases \\
\hline & Dermatomyositis & Lung, breast, colon, pancreas and gynaecological neoplasms \\
\hline & Hypertrophic osteoarthropathy & Lung cancer \\
\hline \multirow{2}{*}{$\begin{array}{l}\text { Paraneoplastic } \\
\text { dermatologic } \\
\text { syndromes }\end{array}$} & Sweet's syndrome & Haematologic malignancies; solid tumours; squamous cell carcinoma \\
\hline & Pemphigus & Non-Hodgkin lymphoma; squamous cell carcinoma \\
\hline
\end{tabular}

renal cell carcinoma and Wilms' tumour, adenocarcinoma and small cell carcinoma of the lung, hepatocellular carcinoma, pancreatic carcinoma and small intestinal adenocarcinomas [9].

A variety of the other hormones may be produced by cancers, for example: luteinizing hormone (LH), placental lactogen (HPL), prolactin (PRL), calcitonin, vasoactive intestinal peptide (VIP), gastrin, neurotensin, somatostatin or glucagon [9].

Paraneoplastic haematologic syndromes. The most common paraneoplastic haematological syndromes are anaemia (haemolytic anaemia or anaemia of chronic disease), leucocytosis, thrombocytopenia or thrombocytosis, coagulapathy (venous thromboembolism, and Trousseau's syndrome, disseminated intravascular coagulation (DIC)) [11, 12, 13].

The pathophysiology of procoagulant changes seems to be connected to a complex phenomenon, including immobilisation, surgery-associated risk, vessel damage through chemotherapeutic agents (chemotherapy and radiation increase the risk of venous thromboembolism), increased levels of procoagulant proteins, reduction of natural anticoagulants (protein $\mathrm{C}$, protein $\mathrm{S}$, antithrombin), suppression of fibrinolytic activity, increased platelet reactivity and activation, enhanced adhesion of neutrophils, down-regulation of thrombomodulin or direct release of procoagulants and cytokines from tumour cells $[14,15]$.
Cancer is the main pathology leading to venous thromboembolism (VTE), developing both deep venous thrombosis (DVT) and pulmonary embolism (PE). Venous thromboembolism (VTE) is one of the major causes of morbidity and mortality in cancer patients. In patients with a first episode of apparently idiopathic thrombosis, $20 \%$ are discovered to already have a malignancy and in up to $34 \%$ a new cancer will be diagnosed within one year [14]. The probability of venous thromboembolism (VTE) in haematological diseases was less than in solid tumors for a long time. However, recent reports suggest that the incidence of thromboembolic events in oncohematological diseases may be similar to that found in solid tumours. The cancer patients suffer from haemorrhage in the case of thrombocytes fluctuations. Patients with the highest 1-year incidence rate of VTE are those with advanced-disease of the brain, lung, uterus, bladder, pancreas, stomach, and kidney [15].

A large clinical trial - Screening for Occult Malignancy in Patients with Symptomatic Idiopathic Venous Thromboembolism (SOMIT) [17] - demonstrated the efficiency of screening in detecting occult cancers. No significant difference was noted in the survival rate.

At present, without definitive evidence showing the advantages in terms of overall survival using invasive diagnostic tests and intensive follow-up, patients should undergo only a thorough physical examination, occult faecal blood test, chest X-ray, a urological visit in men, 
and a gynaecological visit in women. The request for more expensive tests, such as computer tomography (CT) scan, digestive endoscopy or tumour markers should be performed in the case of strong clinical suspicion of occult cancer [15].

Trousseau's syndrome is a cancer-associated hypercoagulable disorder named after the renowned $19^{\text {th }}$ century French physician, Armand Trousseau. It is characterized by warfarin resistance, recurrent superficial thrombophlebitis, both arterial and venous thromboembolism, disseminated intravascular coagulation (DIC) and non-bacterial thrombotic endocarditis. Low molecular weight (LMWH) or unfractionated heparin (UFH), and possibly fondaparinux, are required for successful control of this coagulopathy [16].

Paraneoplastic rheumatic syndromes. Rheumatic patients, particularly rheumatoid arthritis patients, appear to be at higher risk for lymphoma and lung cancer. Patients with cancer are likely to develop paraneoplastic rheumatic syndromes $[18,19,20]$.

Many studies have shown a connection between rheumatic diseases, autoimmunity and cancers. The incidence of occult cancer in patients admitted to a general medical ward during a 10 -year period with rheumatic disorders was $23.1 \%$. The rate of cancer in rheumatic patients is $2.3 \%$ yearly. Several studies have investigated the occurrence of rheumatic symptoms among patients with cancer of certain localization, such as pulmonary, haematopoetic and ovarian [1].

The studies suggest that rheumatic manifestations of occult malignancy include: the rapid onset of an unusual inflammatory arthritis occurring in patients 50 years of age or older, without family history and smokers, test negatively for rheumatoid factor, and the absence of rheumatoid nodules, are major clinical implications for thorough oncological examinations. In the study from Solans-Laque et al., 7.3\% out of malignancy patients reported paraneoplastic rheumatic syndrome [1].

It is generally accepted that the most frequently diagnosed paraneoplastic rheumatic syndromes comprise hypertrophic osteoarthropathy, polyarthritis, dermatomyositis/polymyositis and paraneoplastic vasculitis [1].

The fact that Raynaud's phenomenon in patients above the age of 50, especially those with asymmetric involvement of the fingers or progressive necrosis, can be a paraneoplastic syndrome, preceding the disclosure of cancer, was recognized in the 1970s [21].

Dermatomyositis includes $10 \%$ of the paraneoplastic syndromes. This is an inflammatory myopathy with characteristic cutaneous manifestations. Published studies report a difference in the risk of cancer in patients with dermatomyositis, ranging from $18 \%-45 \%$, and the risk increases with age. The cancers associated with dermatomyositis are of the lung, breast, colon, pancreas and gynaecological [28].

There are many reports of positive ANA (anti-nuclear antibodies) and ENA (extractable nuclear antigens) tests in patients with cancer. The presence of the antibodies characteristic for a rheumatic condition like rheumatoid factor (RF) and antinuclear antibodies (ANA) is frequent, and makes the diagnostic process of the occult malignancy complicated, which sometimes results in delayed diagnosis [1].

Paraneoplastic dermatologic syndromes. Skin pathology can be a marker of internal malignant diseases. The manifestation of skin lesions in patients with cancer is simple in the detection of cancer because the skin is easy to examine and perform a biopsy. Skin can be involved in cancer in different ways: by secondary spread (metastases), as a part of a genetic disorder with a cutaneous component in which there is an inherited predisposition to the later development of malignancy (genodermatoses), as a part of an acquired syndrome due to the toxicity of a carcinogen that induces malignant change and accompanying skin changes, as a consequence of immunosuppression, or by the development of specific lesions that occur as a paraneoplastic syndrome [10].

Many dermatologic conditions, including acrokeratosis paraneoplastica, Sweet's syndrome (acute febrile neutrophilic dermatosis) and paraneoplastic pemphigus have been reported in association with a cancer. Many of the paraneoplastic dermatologic syndromes may precede the clinical diagnosis of a cancer by significant periods of time, and can be of help in anticipating the diagnosis $[10,22]$.

The acrokeratosis paraneoplastica associated with a squamous cell carcinoma was first described in1922, but the name was not given until 1965 by Bazex - Bazex syndrome. The most frequently involved sites of skin lesions are the hands, feet, ears and nose. The skin eruptions consist of squamous hyperkeratotic and erythematous to violaceous lesions. This syndrome is typically associated with cancers of the upper respiratory or digestive tract. Generally, the cutaneous signs appear at the same time as the underlying tumour. Identification of this syndrome leads to the discover a cancer. Treatment of the skin lesions is associated with the suppression of the underlying cancer by surgery, chemotherapy or radiotherapy. Usually, the skin manifestations disappear during the treatment of the tumour [10].

Sweet's syndrome (acute febrile neutrophilic dermatosis) can occur as a paraneoplastic dermatologic syndrome, characterized by the sudden appearance of multiple erythematous nodules and plaques on the skin, leucocytosis and fever. Sweet's syndrome has been associated with haematologic malignancies and solid tumours. About 20\% of the reported patients with Sweet's syndrome have an associated cancer [10].

The Paraneoplastic pemphigus is an autoimmune blistering and erosive mucocutaneous disease associated with cancer, mostly lymphoreticular malignancies [10].

\section{CONCLUSIONS}

When aware of the existence many of paraneoplastic syndromes, it is highly important to correctly pre-diagnose malignant tumors, as well as apply appropriate anti-neoplasm therapy. The paraneoplastic syndromes become clinically important issues, because they may become the first symptom of disease or cause serious complaints. Reducing the intensity of the symptoms of paraneoplastic syndromes can contribute to the improvement of the quality of life of the patient. Primarily, the presence of paraneoplastic syndromes is connected with a worse prognosis, but they can be useful as an indicator of response to treatment.

Knowledge of paraneoplastic syndromes is highly useful in daily clinical practice, and. repeatedly, their appearance is a prelude to recognition of the neoplasm itself. 


\section{REFERENCES}

1.Schiller M, Bohm M, Hensen P, Riemann H, Luger TA, Nashan D. Dermatomyositis associated with malignant melanoma -A marker of poor prognosis? J Am Acad Dermatol, 2006; 54(2): 221-226.

2. Anderson N. Neurologic paraneoplastic syndromes. Journal of Clinical Neuroscience, 2010; 17: 1610-1638.

3. Greenlee JE. Treatment of paraneoplastic neurologic disorders. Neuroimmunology 2010; 12: 212-230.

4. Psimaras D, Carpentier AF, Rossi C. CSF study in paraneoplastic syndromes. J Neurol Neurosurg Psychiatry (in press).

5. Graus F, Delattre JY, Antoine JC, et al. Recommended diagnostic criteria for paraneoplastic neurological syndromes. J Neurol Neurosurg Psychiatry 2004; 75: 1135-1140.

6.Saiz A, Bruna J, Stourac P, et al. Anti-Hu-associated brainstem encephalitis. J Neurol Neurosurg Psychiatry 2009; 80: 404-407.

7. Demarquay G, Didelot A, Rogemond V,Ryvlin P, Gouttard M, Garassus $\mathrm{P}$, et al. Facial pain as first manifestation of anti-Hu paraneoplastic syndrome. J Headache Pain 2010; 11: 355-357.

8.Zilli T, Allal AS. Guillain-Barre syndrome as an atypical manifestation of an esophageal carcinoma. Neurol Sci. 2011; 32: 151-153.

9. De Lellis RA, Xia L. Paraneoplastic endocrine syndromes: a review. Endocrine Pathology 2003; 14(4): 303-117.

10. Toro C, Rinaldo A, Silver CE, Politi M, Ferlito A. Paraneoplastic syndromes in patients with oral cancer. Oral Oncology 2010; 46: 14-18.

11. Staszewski H. Hematological Paraneoplastic Syndromes.Semin. Oncol. 1997; 24: 329-333.

12. Robak T.Zastosowanie czynników wzrostowych układu krwiotwórczego. In: Krzakowski M, ed. Onkologia kliniczna. Warszawa: Borgis Wyd Med. 2001: 343-355 (in Polish).

13. Gorden-Smith EG, Contreras M. Acquired haemolytic anaemia. In: Weatherall DJ, Ledingham JGG, Warrell DA, ed. Oxford Texbook of Medicine. Oksford: Oxford University Press, 1996.p.3547-3548.

14. Piccioli A, Prandoni P. Venous thromboembolism as first manifestation of cancer. Acta Haematol. 2001; 106(1-2): 13-17.

15. Mandal'a M, Falanga R, Roila F. Management of venous thromboembolism (VTE) in cancer patients. ESMO Clinical Practice Guidelines. Annals Oncology 2011; 22: 85-92.
16. Varki A. Trousseau's syndrome: multiple definitions and multiple mechanisms. Blood. 2007; 110(6): 1723-1729.

17. Piccioli A, Lensing AWA, Prins MH. Extensive screening for occult malignant disease in idiopathic venous thromboembolism: a prospective randomized clinical trial. Journal of Thrombosis and Haemostasis 2004; 2 : 884-889.

18. Naschitz JE, Rosner I, Rozenbaum M, et al. Rheumatic Syndromes: Clues to Occult Neoplasia. Semin Arthritis Rheum. 1999; 29: 43-55.

19. Cibere J, Sibley J, Haga M. Rheumatoid arthritis and the risk of malignancy. Arthritis Rheum. 1997; 40: 1580-1586.

20. Tennis E, Andrews E, Bombardier C, et al. Record linkage to conduct an epidemiologic study on the association of rheumatoid arthritis and lymphoma in the province of Saskachewan, Canada. J Clin Epidemiol. 1993; 46: 685-695.

21. DeCross AJ, Sahasrabudhe DM. Paraneoplastic Raynand's phenomenon. Am J Med 1992; 570- 572.

22. Cohen PR, Kurzrock R. Mucocutaneous Paraneoplastic Syndromes. Semin Oncol. 1997; 24: 334-359.

23. Turner HE, Wass JAH. Ectopic Hormone Syndromes. In: DeGroot LJ, Jameson JL, eds. Endocrinology, 4th ed. Philadelphia, PA:WB Saunders, 2001.p.2559-2573.

24. Mundy G, Guise TA. Hypercalcemia of malignancy. Am J Med. 1997; 103: $134-145$.

25. North, WG, Neuropeptide production by small cell carcinoma: vasopressin and oxytocin as plasma markers of disease. J Clin Endocrinol Metab. 1991; 73: 1316-1320.

26. Bliss DP, Battey JF, Linnoila RI, et al. Expression of the atrial natriuretic factor gene in small cell lung cancer/tumors and tumor cell lines. J Natl Cancer Inst. 1990; 82: 305-310.

27. Campling BG, Sarda IP, Baer K, et al. Secretion of atrial natriuretic peptide and vasopressin by small cell lung cancer. Cancer 1995; 75: 2442-2451.

28. Chandiramani M, Joynsony C, Panchaly R, Symondsy RP, Browny LJR, Morgany B, Decatrisz M. Dermatomyositis as a paraneoplastic syndrome in carcinosarcoma of uterine origin. Clinical Oncology 2006; 18: 641-648 